\title{
Influence of Isomaltulose Ingestion on Fat Oxidation During Inclemental Exercise in Endurance Athletes
}

\author{
Satoshi Hattori ${ }^{1}$, Ayaka Noguchi ${ }^{2}$, Katsumi Sasagawa ${ }^{3}$, Hitomi Ogata ${ }^{1,4}$, Masashi Kobayashi ${ }^{2}$, \\ Naomi Omi ${ }^{1, *}$ \\ ${ }^{1}$ Faculty of Health and Sport Sciences, University of Tsukuba, Tsukuba, Japan \\ ${ }^{2}$ Comprehensive Human Sciences, University of Tsukuba, Tsukuba, Japan \\ ${ }^{3}$ Nutraceutical Science Laboratory, Bourbon Institutes of Health, Kashiwazaki, Japan \\ ${ }^{4}$ Research Fellowship for Young Scientists, Japan Society for the Promotion of Science, Tokyo, Japan
}

Email address:

omi.naomign@u.tsukuba.ac.jp (N. Omi)

${ }^{*}$ Corresponding author

\section{To cite this article:}

Satoshi Hattori, Ayaka Noguchi, Katsumi Sasagawa, Hitomi Ogata, Masashi Kobayashi, Naomi Omi. Influence of Isomaltulose Ingestion on Fat Oxidation During Inclemental Exercise in Endurance Athletes. American Journal of Sports Science. Vol. 7, No. 4, 2019 , pp. $193-198$. doi: 10.11648/j.ajss.20190704.20

Received: November 15, 2019; Accepted: December 4, 2019; Published: December 12, 2019

\begin{abstract}
Isomaltulose (ISO) is a carbohydrate (CHO) with metabolic properties that makes it slowly digested and less likely to raise postprandial blood glucose response. We considered that isomaltulose ingestion was difficult to inhibit fat oxidation during incremental exercise. Here we investigated the effect of isomaltulose ingestion on fat oxidation during incremental exercise on a cycle ergometer in endurance athletes $(\mathrm{n}=10)$ who performed an incremental exercise after ISO or sucrose (SUC) ingestion. We measured the fat and $\mathrm{CHO}$ oxidation, blood glucose concentration, and blood lactate concentration of the subjects during the incremental exercise. Between the ISO and SUC groups, the fat oxidation was significantly different at $3 \mathrm{~min}(\mathrm{p}<0.05)$ and CHO oxidation was significantly different at 3,6 , and $12 \min (\mathrm{p}<0.05)$. The ISO group's blood glucose concentrations were significantly lower than those of the SUC group at $-5,3,6,9$, and $12 \min (p<0.05)$. Similarly, the ISO group's blood lactate concentrations were significantly lower than those of the SUC group at $-5,0,3,6,9$, and $18 \mathrm{~min}(\mathrm{p}<0.05)$. These results indicate that isomaltulose ingestion causes only slight fat oxidation inhibition and a slow increase in blood lactate levels compared with sucrose ingestion by a gradual rise in the blood glucose level.
\end{abstract}

Keywords: Isomaltulose, Endurance Exercise, Fat Oxidation, Carbohydrate Oxidation

\section{Introduction}

The fat and $\mathrm{CHO}$ substrates are used for adenosine triphosphate (ATP) synthesis through the tricarboxylic acid cycle and the electron transport chain in mitochondria in the cytosol. CHO depletion frequently occurs in endurance exercise [1]. In fact, endurance exercise causes a significant loss of $\mathrm{CHO}$ substrates in various organs (e.g., muscle glycogen and blood glucose) [2,3]. CHO depletion leads to a remarkable decrease in endurance exercise performance [2]. It is thus generally known that saving $\mathrm{CHO}$ oxidation and an enhancement of fat oxidation are necessary to maintain an animal or human's endurance exercise capacity, because the $\mathrm{CHO}$ energy storage is lower than fat energy storage.
It is recognized that $\mathrm{CHO}$ ingestion during prolonged exercise promotes endurance exercise performance [4]. This effect of $\mathrm{CHO}$ ingestion on endurance exercise capacity has been attributed to a retard of the decrease in $\mathrm{CHO}$ accumulation during endurance exercise. On the other hand, $\mathrm{CHO}$ ingestion promotes $\mathrm{CHO}$ oxidation [5], but it inhibits fat oxidation during exercise [6]. Therefore, it is suitable a kind of $\mathrm{CHO}$ ingestion that does not strongly promote $\mathrm{CHO}$ oxidation and slightly inhibits fat oxidation to increase endurance exercise capacity.

The carbohydrate isomaltulose (6- $O$-D-glucopyranosyl-Dfructose, or ISO) is a structural isomer of sucrose. Previous studies demonstrated that ISO was digested slowly compared to sucrose (by fivefold) though it provides energy $(4 \mathrm{kcal} / \mathrm{g}$ ) equivalent to that provided by sucrose [7]. Thus, ISO does 
not cause a rapid increase of blood glucose levels after ingestion compared to sucrose [8,9].

It was reported that during endurance exercise, the oxidation of $\mathrm{CHO}$ and the blood insulin response after ISO ingestion change more slowly compared to after sucrose ingestion [10], which indicated that ISO ingestion modestly increases $\mathrm{CHO}$ oxidation and weakly decreases fat oxidation. However, that study evaluated only the influence of ISO ingestion on constant-intensity exercise. It is widely accepted that fat oxidation greatly changes depending on the intensity of exercise [11-13]. The effects of ISO ingestion on highversus low-intensity endurance exercise have not been established.

The sine (SIN) model is a mathematical model used to describe fat oxidation kinetics during incremental exercise [14]. This mathematical model can estimate the characteristics of fat oxidation kinetics in order to determine independent variables (i.e., dilatation, symmetry, and translation) using the SIN model. Chenevière reported that the fat oxidation during graded exercise after a submaximal incremental test was higher than the same exercise in the case of no test [15]. We speculated that the SIN model could be sued to calculate the effect of $\mathrm{CHO}$ ingestion on fat oxidation kinetics during incremental exercise. In fact, we demonstrated that other $\mathrm{CHO}$ material affected on fat oxidation in endurance athletes during exercise due to this model [16].

We conducted the present study to investigate fat oxidation and fat oxidation kinetics after ISO ingestion during incremental exercise. We hypothesized that if we would observe a slight inhibition of fat oxidation during exercise due to ISO ingestion compared with SUC ingestion, ISO may be considered a suitable $\mathrm{CHO}$ to use during endurance exercise.

\section{Methods}

\subsection{Subjects}

Ten male endurance athletes (long distance runners and triathletes) participated in this study. They were recruited to participate in this study after giving their written informed consent. This study was approved by the Ethics Committee of the University of Tsukuba (Faculty of Health and Sport Sciences, 24-2), and it was conducted in accordance with the Declaration of Helsinki. These subjects didn't have any pathological conditions, and they didn't intake any supplements and medications.

\subsection{Preliminary Training}

Before the start of the experiments, the subjects were familiarized with the equipment and the procedures. The subjects tried a pedaling exercise on an electromagnetically braked cycle ergometer before the approximately of the experimental trials (PowerMax-VII, Combi Wellness, Tokyo).
Table 1. The physiological characteristics of the subjects.

\begin{tabular}{ll}
\hline Physiological parameters & \\
\hline Age & $20.6 \pm 2.5$ \\
Height $(\mathrm{cm})$ & $172.3 \pm 5.2$ \\
Body weight $(\mathrm{kg})$ & $57.7 \pm 4.6$ \\
Body mass index $\left(\mathrm{kg} / \mathrm{m}^{2}\right)$ & $19.4 \pm 0.9$ \\
Body fat $(\%)$ & $10.6 \pm 2.1$ \\
VO 2 max $(\mathrm{mL} / \mathrm{kg} / \mathrm{min})$ & $62.7 \pm 5.8$ \\
HRmax (beat $/ \mathrm{min})$ & $174.3 \pm 18.7$ \\
Resting blood glucose level $(\mathrm{mg} / \mathrm{dL})$ & $95.0 \pm 13.1$ \\
Resting blood lactate level $(\mathrm{mM})$ & $0.89 \pm 0.40$ \\
\hline
\end{tabular}

Ṿalues are mean \pm SD.

$\mathrm{VO}_{2}$ max: maximum oxygen uptake, HR: Heart rate.

\subsection{Prestudy Evaluation}

We estimated the body composition of subjects due to bioelectrical impedance analyzer (MC-190 SV, TANITA).

To evaluate of each subject's maximum oxygen uptake (V $\mathrm{O}_{2 \max }$ ), we carried out $\mathrm{VO}_{2 \max }$ test for the subjects on the electromagnetically braked cycle ergometer at least 1 week before the ingestion experiment [17]. In this test, these subjects exercised warm-up at $1 \mathrm{kp}(60 \mathrm{rpm}, 5 \mathrm{~min})$ before maximum cycling exercise. After this period, they tried the first stage of the test at $2.5 \mathrm{kp}(60 \mathrm{rpm}, 3 \mathrm{~min})$, and it was increased by $0.1 \mathrm{kp} / \mathrm{min}(60 \mathrm{rpm})$ until exhausted exercise level. We measured the heart rate (HR) of the subjects due to HR monitor (S610i, Polar, Tokyo). Moreover, we recorded the components $\left(\mathrm{V} \mathrm{O}_{2}\right.$ and $\left.\mathrm{VCO}_{2}\right)$ of their breath gases (averaged data for the last $1 \mathrm{~min}$ of each stage) due to breathby-breath measurements by an aero-monitoring system (AE310-S, Minato Medical Science Co., Tokyo) to evaluate their respiratory functions throughout the test. We calibrated the gas analyzers with gases of known concentrations $(5 \%$ $\mathrm{CO}_{2}$ and $15 \% \mathrm{O}_{2}$ ), and the volume was automatically calibrated at the flow rate of $2 \mathrm{~L} \cdot \mathrm{s}^{-1}$ before each test. We assessed the maximal oxygen uptake $\left(\mathrm{V} \mathrm{O}_{2}\right)$ when the parameters were satisfied conditions at least two of the following three criteria. Namely, (1) a leveling off of $\mathrm{VO}_{2}$ (defined as an increase of no more than $2 \mathrm{ml} \cdot \mathrm{kg}^{-1} \cdot \mathrm{min}^{-1}$ ) during the exercise test, (2) an HR value exceeding $90 \%$ of the age-predicted maximal value, and (3) a respiratory exchange ratio (RER) greater than 1.1. We maintained the temperature at $22 \pm 1^{\circ} \mathrm{C}$ during the testing in the laboratory room.

\subsection{Diets and Physical Activity the Day Before Testing}

To control the individual energy and nutrition balances, we gave the subjects' diets based on dietary reference intakes for Japanese [18] on the day before the experiment. In particular, the diets (15\% protein, $25 \%$ fat and $60 \%$ carbohydrate) were decided to reach an their energy balance assuming the subject's resting metabolic rate to be $24.0 \mathrm{kcal} \mathrm{kg}^{-1} \mathrm{day}^{-1}$ and his physical activity factor to be 1.75 . Physical activity was also controlled to a normal level on the previous day of experiment. 


\subsection{Experimental Design}

This study was carried out a randomized crossover design of the subjects. There were wash-out periods at least 1 week between two experimental sessions, namely a session after ISO ingestion and the other session after SUC ingestion. In order to exclude factors of circadian rhythm, these tests were performed at the same time in the morning. Moreover, they also came to the laboratory on two occasions after a 10- to 12-h overnight fasting condition since dinner on the previous day to rule out food intakes. They were equipped with a heart rate monitor at the time of arriving at the laboratory. The subjects drank a beverage containing $8 \%$ sucrose (SUC) or $8 \%$ isomaltulose (ISO) per $500 \mathrm{ml}$ water before $15 \mathrm{~min}$ at the start of warm-up periods in each experiment. We used the incremental exercise protocol based on Chenevière's test to evaluate fat oxidation kinetics [14]. After the subjects exercised 5-min warm-up period $(0.7 \mathrm{kp}, 60 \mathrm{rpm})$ on a cycle ergometer, the work rate was increased by $0.3 \mathrm{kp}$ per $3 \mathrm{~min}$ until $\mathrm{RER}=1.0$ without a rest $(60 \mathrm{rpm}$ at all stages $)$. Breath gases were collected by the aero-monitoring system during the incremental exercise after the gas analyzers were calibrated. These gases data $\left(\mathrm{VO}_{2}, \mathrm{VCO}_{2}\right)$ for the last $1 \mathrm{~min}$ of each stage were averaged. We collected blood samples (150 $\mu \mathrm{l})$ using a Safety lancet (Becton, Dickinson, Tokyo) and a capillary tube (Terumo, Tokyo) during the last $15 \mathrm{sec}$ of each stage.

\subsection{Analysis}

We analyzed the blood samples immediately. The blood lactate concentrations were determined using a lactate analyzer (1500 Sport, YSI, Yellow Springs, OH). The blood glucose concentrations were measured by a small electrodetype glucose analyzer (Antsense III, Horiba, Fukuoka, Japan).

\subsection{Calculations}

The total $\mathrm{CHO}$ and fat oxidation rates were calculated from the average values of $\mathrm{VO}_{2}$ and $\mathrm{CO}_{2}(\mathrm{~L} / \mathrm{min})$ during the last 1 minute of every stage until $\mathrm{RER}=1.0$. These were calculated using mathematical equations (i.e. $\mathrm{CHO}$ oxidation rate $\left(\mathrm{g} \cdot \mathrm{min}^{-1}\right)=4.55 . \mathrm{VCO}_{2}-3.21 \mathrm{VO}_{2}$, and Fat oxidation rate $\left.\left(\mathrm{g} \cdot \mathrm{min}^{-1}\right)=1.67 \mathrm{VO}_{2}-1.67 \mathrm{VCO}_{2}\right)$ [19].

We used the SIN model to calculate the fat oxidation kinetics in the experiment periods [20]. This mathematical model is able to estimates the fat oxidation kinetics based on the calculation of three independent variables (Dilatation, Symmetry, and Translation). In addition, Three characteristics of the fat oxidation kinetics were simultaneously calculated, namely the maximum fat oxidation (MFO, the maximum fat oxidation during incremental exercise), the Fat ${ }_{\max }$ (the exercise intensity at the MFO) [11], and the $\mathrm{Fat}_{\text {min }}$ (the exercise intensity at the minimum value of fat oxidation).

\subsection{Statistics}

Experimental data were expressed as means \pm SD. Statistical analyses were calculated using SPSS statistical software (Version 20, IBM Japan, Tokyo, Japan). A comparison of variables between two conditions was performed by a student's $t$ test for paired samples. The Holm post hoc test was used for two-way ANOVA using R (version 3.6.1, R Development Core Team, Vienna, Austria). All statistical significance was accepted at $P<0.05$.

\section{Results}

\subsection{Subject Characteristics}

The physical parameters of the subjects are showed (Table. 1). The $\mathrm{VO}_{2 \max }$ and $\mathrm{HR}_{\max }$ of subjects were measured in a pre-study evaluation.

\subsection{Fat and Carbohydrate Oxidation}

We examined the effect of ISO ingestion on fat oxidation in the 10 endurance athletes. The results demonstrated a significant difference in fat oxidation at $3 \mathrm{~min}$ between the ISO and SUC ingestion $(p<0.05)$. The fat oxidation values after ISO ingestion were high compared to SUC at 6-15 min (Figure 1A). There were no other significant differences in fat oxidation between the SUC and ISO ingestions at 18-24 min. The results of two-way ANOVA were as follows: Beverage $(p<0.05)$, Time (not significant, n.s.), Beverage $x$ Time interaction (n.s.). There were no differences in iAUC of the Fat oxidation after the SUC versus ISO ingestion (Figure 2A).

In contrast, the $\mathrm{CHO}$ oxidation values after ISO ingestion were low compared to those after SUC ingestion during the incremental exercise (Figure 1B). There were significant differences between SUC and ISO at 3, 6, and $12 \mathrm{~min}$ $(p<0.05)$. The results of two-way ANOVA were as follows: Beverage $(p<0.001)$, Time $(p<0.001)$, Beverage $x$ Time interaction (n.s.). There were no differences in iAUC of the Fat oxidation after the SUC versus ISO ingestion (Figure 2B).

Table 2. The SIN model's independent variables and characteristics of fat oxidation kinetics.

\begin{tabular}{lll}
\hline & SUC & ISO \\
\hline Dilatation & $-0.05 \pm 0.51$ & $-0.36 \pm 0.29$ \\
Symmetry & $1.05 \pm 0.05$ & $0.95 \pm 0.19$ \\
Translation & $0.21 \pm 0.62$ & $0.12 \pm 0.26$ \\
MFO & $0.17 \pm 0.10$ & $0.25 \pm 0.09$ \\
Fatmax & $44.5 \pm 20.5$ & $44.9 \pm 12.5$ \\
Fatmin & $91.9 \pm 18.6$ & $84.9 \pm 16.7$ \\
\hline
\end{tabular}

Values are mean \pm SD. MFO: Maximam fat oxidation during incremental exercise. Fat $_{\max }$ : exercise intensity at the MFO. Fat ${ }_{\text {min }}$ : exercise intensity at the minimum value of fat oxidation. 

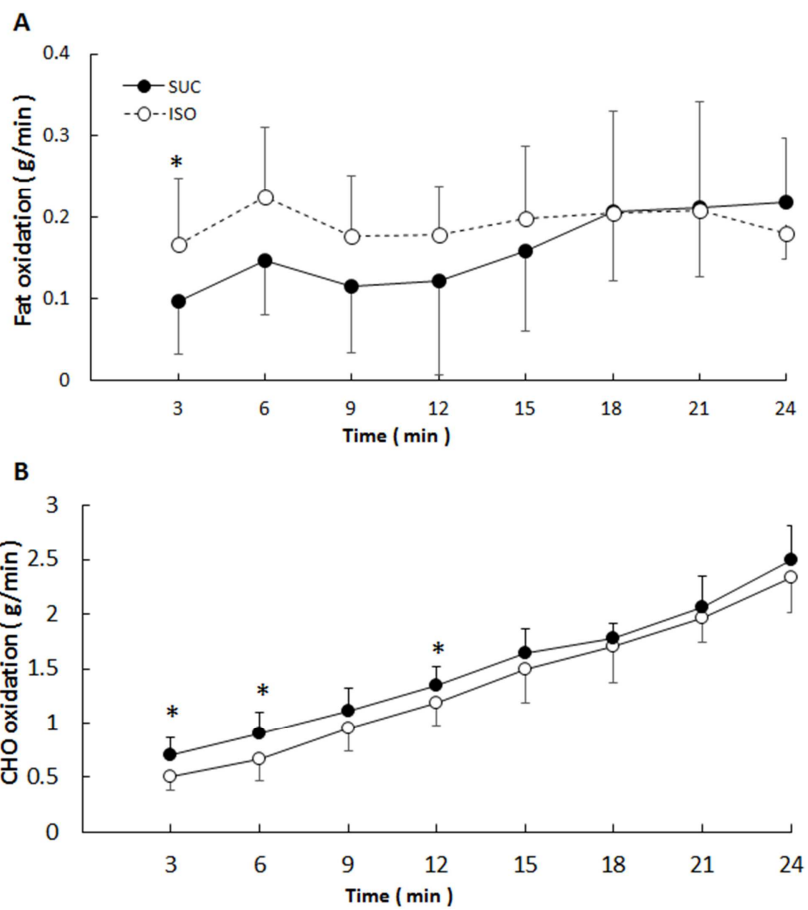

Figure 1. Fat oxidation and carbohydrate (CHO) oxidation.

Fat oxidation (A) and $\mathrm{CHO}$ oxidation (B) during incremental exercise in the endurance athletes $(\mathrm{n}=10)$. SUC (sucrose group), ISO (isomaltulose group). Values are means \pm SD. ${ }^{*} \mathrm{p}<0.05$ SUC group vs. ISO group. The results of fat oxidation by two-way ANOVA were as follows: Beverage $(\mathrm{p}<0.05)$, Time (n.s.), Beverage $\mathrm{x}$ Time interaction (n.s.). The results of $\mathrm{CHO}$ oxidation by two-way ANOVA were as follows: Beverage $(\mathrm{p}<0.001)$, Time $(\mathrm{p}<0.001)$, Bevarage $\mathrm{x}$ Time interaction (n.s.).

\subsection{Fat Oxidation Kinetics and the Characteristics of Fat Oxidation Kinetics}

A

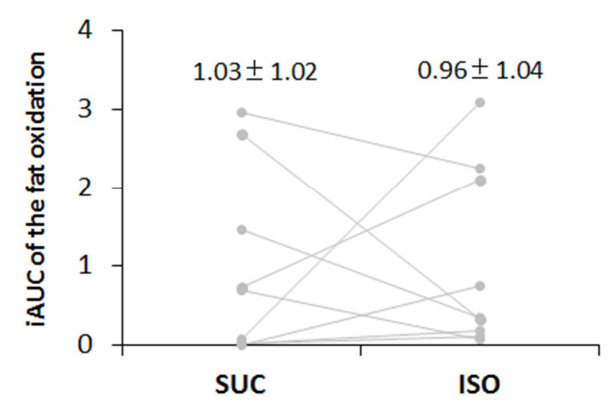

B

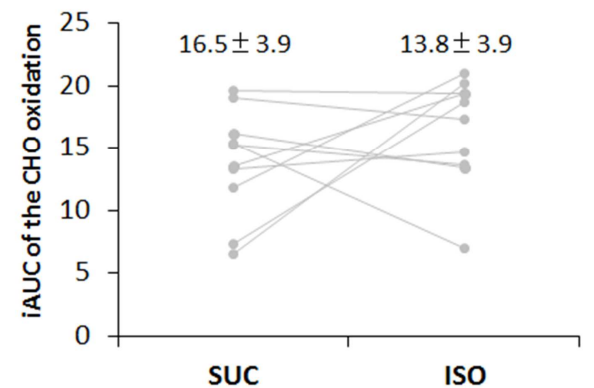

Figure 2. The iAUC of fat oxidation and $\mathrm{CHO}$ oxidation at each stage.
We calculated independent values of the SIN model (dilatation, symmetry, and translation) to evaluate the changes in fat oxidation kinetics due to ISO ingestion (Table 2). The results revealed no significant differences in the independent values between ISO and SUC. Similarly, there were no significant differences in the characteristics of fat oxidation kinetics (MFO, Fat ${ }_{\max }$, and $\mathrm{Fat}_{\min }$ ) (Table 2).

The iAUC of the fat oxidation (A) and the iAUC of the $\mathrm{CHO}$ oxidation (B) at each stage during incremental exercise in the endurance athletes $(n=10)$. Values are means \pm SD. ${ }^{*} \mathrm{p}<0.05$ SUC vs. ISO group.

\subsection{Blood Glucose Concentration}

Blood glucose concentrations in ISO were lower than these in SUC during all exercise periods (Figure 3A). These in ISO were significantly lower than these in SUC $(p<0.05$ and $\mathrm{p}<0.01 ;-5,3,6,9,12 \mathrm{~min})$. The results of two-way ANOVA were as follows: Beverage $(p<0.001)$, Time $(p<0.001)$, Beverage $x$ Time interaction (n.s.).

\subsection{Blood Lactate Concentration}

The ISO ingestion resulted in the maintenance of lower blood lactate concentrations compared to SUC at rest and during the warm-up and incremental exercise periods (Figure 3B). There were significantly differences between ISO and SUC ( $p<0.05$ and $p<0.01 ;-5,0,3,6,9$, and $18 \mathrm{~min})$. The results of two-way ANOVA were as follows: Beverage $(p<0.001)$, Time $(p<0.001)$, Beverage $x$ Time interaction (n.s.).
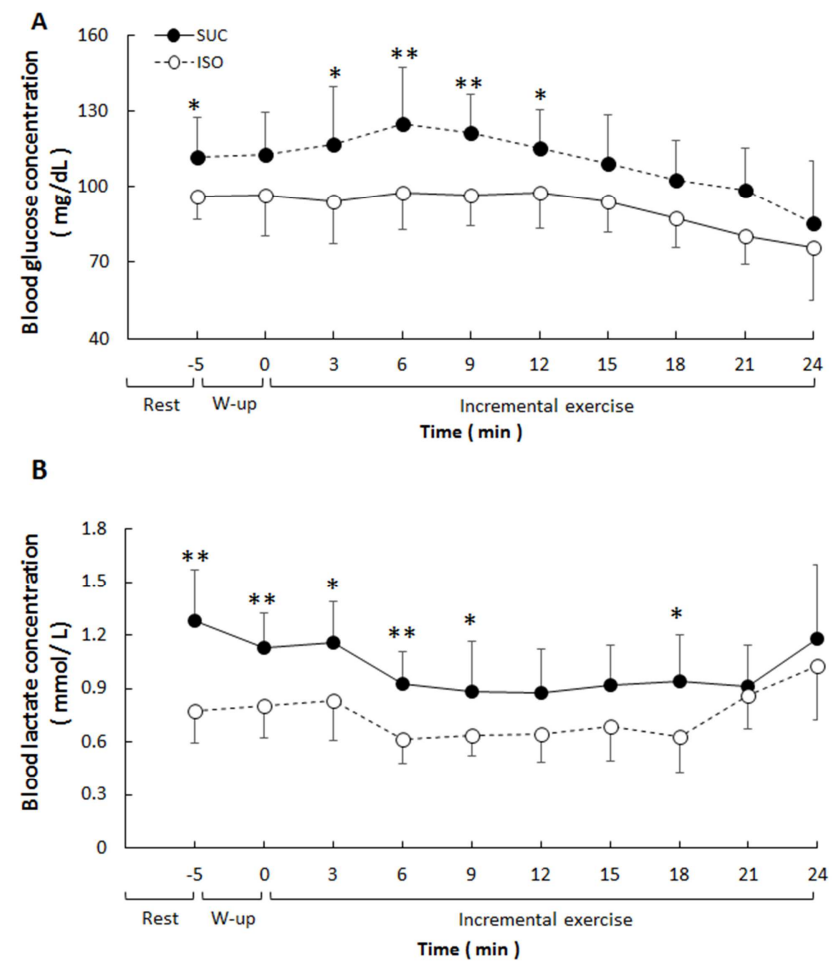

Figure 3. Blood glucose concentration and blood lactate concentration.

The blood glucose concentrations (A) and blood lactate 
concentration (B) during incremental exercise in endurance athletes $(\mathrm{n}=10)$. SUC (sucrose group), ISO (isomaltulose group). $-5 \mathrm{~min}$; Rest period before w-up (warming up period), $-5 \mathrm{~min} \sim 0 \mathrm{~min}$; w-up, from $0 \mathrm{~min}$; incremental exercise period. Values are means \pm SD. ${ }^{*} \mathrm{p}<0.05,{ }^{* *} \mathrm{p}<0.01$ SUC vs. ISO group. The results of the blood glucose concentration by two-way ANOVA were as follows: Beverage $(p<0.001)$, Time $(p<0.001)$, Beverage $x$ Time interaction (n.s.). The results of the blood lactate concentration by two-way ANOVA were as follows: Beverage $(p<0.001)$, Time $(p<0.001)$, Beverage $x$ Time interaction (n.s.).

\section{Discussion}

We investigated the effect of ISO ingestion on fat oxidation and $\mathrm{CHO}$ oxidation kinetics during incremental exercise in endurance athletes. We found that the fat oxidation in the ISO group was higher than that in the SUC group, and the CHO oxidation in the ISO group was lower than that in the SUC group during exercise especially in lowto moderate-intensity $(\sim 15 \mathrm{~min})$. These results indicated that ISO ingestion did not strongly promote $\mathrm{CHO}$ oxidation and slightly inhibited the fat oxidation at the low- and moderateintensity exercise.

It is generally recognized that $\mathrm{CHO}$ ingestion increases $\mathrm{CHO}$ oxidation and decreases fat oxidation $[5,6]$. In the present study, the ISO group showed high levels of fat oxidation compared to the SUC group (Figure 1A). And, the $\mathrm{CHO}$ oxidation in the ISO group was lower than that in the SUC group (Figure 1B). A previous study reported that ISO ingestion leads to a slow up-regulation of $\mathrm{CHO}$ oxidation and a weak down-regulation of fat oxidation in exercised subjects [10]. Thus, our present findings also indicate that ISO has little effect on fat and $\mathrm{CHO}$ oxidation during low- and moderate-intensity exercise.

On the other hand, there were no significantly differences in the fat or CHO oxidation between the SUC and ISO groups after $15 \mathrm{~min}$ (Figure 1A, 1B). It is well understood that fat and $\mathrm{CHO}$ metabolism are dependent on exercise intensity [7-9]. In fact, fat oxidation is highest in moderateintensity exercise, and it almost abolish near lactate threshold levels during exercise [21]. Therefore, our present results may indicate that the ISO ingestion did not affect these metabolisms in the high-intensity exercise because $\mathrm{CHO}$ may be preferential metabolized at these exercise intensity.

In contrast, there were also no significantly differences in the iAUC of fat or $\mathrm{CHO}$ oxidation, and there were individual differences in these parameters (Figure 1B, 2B). It was reported that fat and $\mathrm{CHO}$ oxidation was dependent on the exerciseassociated oxidative capacity [22]. There are also individual differences at the $\mathrm{VO}_{2 \max }$, namely exercise-associated oxidative capacity of subjects in this study (minimum value: 52.0, maximum value: 69.0). These results may be caused by oxidative capacity during exercise in these subjects.

The blood glucose concentrations after ISO ingestion were always lower compared to those after SUC ingestion during the incremental exercise (Figure 3A). Previous studies were reported that ISO ingestion more slowly elevated in the blood glucose concentration than that in SUC ingestion [8, 23]. The blood glucose levels that we observed in the present study indicate that ISO ingestion also slowly increase the blood glucose during incremental exercise.

Acute $\mathrm{CHO}$ ingestion causes an increase in the blood lactate level $[24,25]$. In this study, the blood lactate levels after ISO ingestion were lower than those after SUC ingestion, from $-5 \mathrm{~min}$ to $18 \mathrm{~min}$ (Figure. $3 \mathrm{~B}$ ). These results indicate that the subjects' blood lactate levels were influenced by absorption rate differences between isomaltulose and sucrose. However, there were no differences in blood lactate levels between the SUC and ISO groups after $21 \mathrm{~min}$ (Figure $3 \mathrm{~B}$ ). Blood lactate is produced through a glycolytic pathway in exercise. Our findings suggest that the blood lactate production was elevated by not only $\mathrm{CHO}$ ingestion but also the increase in the exercise intensity, thereby resulting a no significant difference in blood lactate between the two groups after $21 \mathrm{~min}$. Thus, isomaltulose ingestion may affect the blood lactate concentration during low- and moderateintensity exercise, while it may not affect this concentration during high-intensity exercise.

We also observed that the fat oxidation kinetics was not influenced by ISO ingestion (Tables 1, 2). It was reported that 1-hr continuous exercise resulted in significant differences in fat oxidation kinetics in graded exercise compared to no continuous exercise [15]. These results might be affected by $\mathrm{CHO}$ digestion due to the $1 \mathrm{hr}$ of continuous exercise. In contrast, our present findings did not reveal any significant differences in fat oxidation kinetics between the ISO ingestion and $\mathrm{CHO}$ ingestion. Our results indicate that ISO ingestion does not cause a notable difference in $\mathrm{CHO}$ accumulation, as continuous exercise does.

In conclusion, the ingestion of isomaltulose did not strongly contributed to the promotion of $\mathrm{CHO}$ oxidation and slightly inhibited fat oxidation compared with that of sucrose during incremental exercise by endurance athletes.

\section{Conclusion}

In addition, the glucose consentrations in ISO were lower than these in SUC, similary in the blood lactate concentrations. These findings indicates that ISO ingestion contribute to endurance athletes to slightry inhibit in fat oxidation and slowry increase in $\mathrm{CHO}$ oxidation during incremental exercise compared with SUC ingestion.

\section{Conflict of Interest}

All the authors do not have any possible conflicts of interest.

\section{References}

[1] ALTLAND PD, HIGHMAN B. 1961. Effects of exercise on serum enzyme values and tissues of rats. Am J Physiol. 201: 393-5. 
[2] Bergström J, Hermansen L, Hultman E, Saltin B. 1967. Diet, Muscle Glycogen and Physical Performance. Acta Physiol Scand. 71 (2): 140-50.

[3] Carlson LA, Froeberg S, Persson S. 1965. Concentration and turnover of the free fatty acids of plasma and concentration of blood glucose during exercise in horses. Acta Physiol Scand. 63: 434-41.

[4] Jeukendrup AE, Jentjens R. 2000. Oxidation of carbohydrate feedings during prolonged exercise: current thoughts, guidelines and directions for future research. Sports Med. 29 (6): 407-24.

[5] Jeukendrup AE. 2004. Carbohydrate intake during exercise and performance. Nutrition. 20 (7-8): 669-77.

[6] Horowitz JF, Mora-Rodriguez R, Byerley LO, Coyle EF. 1997. Lipolytic suppression following carbohydrate ingestion limits fat oxidation during exercise. Am J Physiol. 273 (4 Pt 1): E768-75.

[7] Tsuji Y, Yamada K, Hosoya N, Moriuchi S. 1986. Digestion and absorption of sugars and sugar substitutes in rat small intestine. J Nutr Sci Vitaminol (Tokyo). 32 (1): 93-100.

[8] Kawai K, Okuda Y, Yamashita K. 1985. Changes in blood glucose and insulin after an oral palatinose administration in normal subjects. Endocrinol Jpn. 32 (6): 933-6.

[9] Holub I, Gostner A, Theis S, Nosek L, Kudlich T, Melcher R, Scheppach W. 2010. Novel findings on the metabolic effects of the low glycaemic carbohydrate isomaltulose (Palatinose). $\mathrm{Br} J$ Nutr. 103: 1730-7.

[10] Achten J, Jentjens RL, Brouns F, Jeukendrup AE. 2007. Exogenous oxidation of isomaltulose is lower than that of sucrose during exercise in men. J Nutr. 137: 1143-8.

[11] Brooks GA, Mercier J. 1994. Balance of carbohydrate and lipid utilization during exercise: the "crossover" concept. $J$ Appl Physiol (1985). 76 (6): 2253-61.

[12] Romijn JA, Coyle EF, Sidossis LS, Gastaldelli A, Horowitz JF, Endert E, Wolfe RR. 1993. Regulation of endogenous fat and carbohydrate metabolism in relation to exercise intensity and duration. Am J Physiol. 265 (3 Pt 1): E380-91.

[13] van Loon LJ, Greenhaff PL, Constantin-Teodosiu D, Saris WH, Wagenmakers AJ. 2001. The effects of increasing exercise intensity on muscle fuel utilisation in humans. $J$ Physiol. 1; 536 (Pt 1): 295-304.
[14] Chenevière X, Malatesta D, Peters EM, Borrani F. 2009. A mathematical model to describe fat oxidation kinetics during graded exercise. Med Sci Sports Exerc. 41 (8): 1615-25.

[15] Chenevière X, Borrani F, Ebenegger V, Gojanovic B, Malatesta D. 2009. Effect of a 1-hour single bout of moderate-intensity exercise on fat oxidation kinetics. Metabolism. 58: 1778-86.

[16] Hattori S, Noguchi A, Ogata H, Kobayashi M, Omi N. 2019. The Effect of Maple Syrup Ingestion on Fat Oxidation During Incremental Exercise in Endurance Athletes. Am J Spo Sci. 7 (4): 149-154.

[17] Chin LM, Kowalchuk JM, Barstow TJ, Kondo N, Amano T, Shiojiri T, Koga S. 2011. The relationship between muscle deoxygenation and activation in different muscles of the quadriceps during cycle ramp exercise. J Appl Physiol (1985). 111 (5): 1259-65.

[18] Ministry of Health, Labour, and Welfare of Japan. 2005. Dietary reference intakes for Japanese. Tokyo: DaiichiShuppan.

[19] Frayn KN. 1983. Calculation of substrate oxidation rates in vivo from gaseous exchange. $J$ Appl Physiol Respir Environ Exerc Physiol. 55 (2): 628-34.

[20] Chenevière X, Borrani F, Sangsue D, Gojanovic B, Malatesta D. 2011. Gender differences in whole-body fat oxidation kinetics during exercise. Appl Physiol Nutr Metab. 36: 88-95.

[21] Achten J, Jeukendrup AE. 2003. Maximal fat oxidation during exercise in trained men. Int J Sports Med. 24 (8): 603-8.

[22] Overmyer KA, Evans CR, Qi NR, Minogue CE, Carson JJ, Chermside-Scabbo CJ, Koch LG, Britton SL, Pagliarini DJ, Coon JJ, Burant CF.. 2015. Maximal oxidative capacity during exercise is associated with skeletal muscle fuel selection and dynamic changes in mitochondrial protein acetylation. Cell Metab. 3; 21 (3): 468-78.

[23] Kawai K, Yoshikawa H, Murayama Y, Okuda Y, Yamashita K. 1989. Usefulness of palatinose as a caloric sweetener for diabetic patients. Horm Metab Res. 21: 338-40.

[24] HUCKABEE WE. 1958. Relationships of pyruvate and lactate during anaerobic metabolism. I. Effects of infusion of pyruvate or glucose and of hyperventilation. J Clin Invest. 37: 244-54.

[25] Doar JW, Cramp DG, Maw DS, Seed M, Wynn V. 1970. Blood pyruvate and lactate levels during oral and intravenous glucose tolerance tests in diabetes mellitus. Clin Sci. 39: 259-69. 